\title{
Hospital Indoor Navigation System Based on QR Codes
}

\author{
You Zhou* \\ School of Taizhou, Nanjing Normal University, Taizhou 225300, Jiangsu Province, China \\ *Corresponding author: You Zhou, 550569316@qq.com
}

\begin{abstract}
With the continuous increase in China's population, the number of people visiting hospitals are also increasing. In order to meet the needs of patients, hospitals are expanding their scale, and departments are becoming more and more sophisticated. While improving the quality of medical services, it has become more difficult for patients to seek medical consultation. In order to improve the quality of medical services, a indoor navigation system based on QR code has been designed for hospitals, which realizes indoor navigation by scanning a QR code. This low-cost technology has accurate positioning, which brings convenience in locating and navigating departments.
\end{abstract}

Keywords: QR code; Indoor navigation; WeChat platform

Publication date: November 2021; Online publication: November 30, 2021

\section{Introduction}

\subsection{Research background}

With the continuous progress of science and technology, the reform of medical and health system, as well as the transformation of the mode of hospital service, hospitals are constantly developing in the direction of convenience and fast information. This means that the number of patients is growing. At the same time, hospitals are also expanding, the medical specialty is subdivided, the division of labor of various clinical specialties is more detailed, the setting of departments is more refined, and many new hospitals are progressing. The design of a reasonable hospital navigation system can help patients and visitors find relevant departments quickly, efficiently, and accurately, reduce medical difficulty, as well as improve their medical service experience. This has become the focus of attention.

The outpatient volume of general hospitals is generally large. For patients visiting the hospital for the first time, facing the complex and strange hospital environment ${ }^{[1]}$, a considerable amount of time is needed for them to find the department they wish to go to. For example, Nanjing Gulou Hospital has 32 clinical departments alone. For large local hospitals, it is not uncommon for the building area of a single building to reach or even exceed 500,000 square meters. Without reliable guidance, it is difficult for patients to find departments, clinics, and examination rooms in such a huge building. It is easy to fall into "turbulence" and waste valuable time, and in worst case scenario, miss the best treatment time, which will greatly aggravate the anxiety of patients and their families as well as the tension between doctors and patients.

The classification of departments not only increases the burden on patients, but also reduces the efficiency of doctors, and objectively increases the possibility of tension between doctors and patients. In view of this situation, there are two traditional medical guidance methods. The first is to set up a medical guidance platform, but because the medical guidance platform is fixed, it is not convenient for patients to find it in real time. The second is to set up a plane guidance diagram, but the amount of information storage is limited; hence, with poor real-time sense, it is not easy to identify the direction. 
With the popularization and development of mobile network, mobile phones have become an indispensable communication tool for people's life, work, study, and communication, and WeChat has undertaken the leading role of mobile phone software applications. By the end of 2018, the average daily number of WeChat transmissions was 38 billion, with users in more than 200 countries and nearly 20 languages around the world. As an important part of WeChat, WeChat public platform has quickly penetrated into all walks of life with various advantages, including convenience, short development time, and low cost. The use of WeChat public platform is not only convenient and timely, but also widely applicable ${ }^{[1]}$.

QR codes have a large amount of information storage, a wide range of coding, strong error correction function, high reliability, and low production cost compared with other types of technologies. Therefore, saving a location information into a QR code can improve the feasibility of the system.

With this technology, the department information is bound to the QR code. Users can then easily scan these QR codes for positioning. With the navigation function, the nearest QR code can be used to locate the location of the person and find one's destination. The system can accurately locate all departments of the hospital. This technology easily guides patients around the hospital and improves their satisfaction ${ }^{[2]}$.

\subsection{Steps and contents}

QR codes can be placed at eye-catching areas in each building and department. The specific positions and other information can also be stored in QR codes.

In order to realize indoor department navigation on the basis of accurate positioning, the corresponding information on the department location of each QR code needs to be stored in the database. When users have determined their own location coordinates, they can then scan the nearest QR code. After determining the target location information, the system retrieves the route map stored in the database in advance. Through the intuitive display of WeChat client, users can then find the destination according to the route shown in the map ${ }^{[3]}$.

There are several steps in the development process of the system.

\subsubsection{Preparations before development}

(1) Investigate and analyze the hospital's treatment business process and user needs.

(2) Apply for WeChat public platform account and external network server that needs data interaction to build the environment for system operation.

\subsubsection{Demand analysis and design}

This step mainly includes the following aspects: system demand side analysis, functional demand analysis, system interface design, WeChat client design, background database design, etc.

\subsubsection{System design and implementation}

Several frameworks and technologies are used in the design and implementation of the system, including Bootstrap, WeChat, JavaScript-SDK interface, JavaScript chart library, Highcharts, amCharts, h-ui frontend framework, jQuery, and indoor positioning technology (IPS).

\section{System design}

\subsection{Overall design of the system}

In the WeChat public platform development document, when creating a custom menu, two interfaces are required, both of which are HTTP requests. First, encapsulate the menu. The request method is as follows: 
(1) receive HTTP request layer - handle HTTP request function, different types of messages, and messages in XML format;

(2) distribution layer - achieve scalability and expand the system at any time;

(3) combine with business processing and data logic layer.

The system requires the user to confirm the user's current location by scanning the nearest QR code through the QR code scanning applet. During navigation, the navigation route is planned according to the starting point and destination entered by the user, and the route map is fed back to the user to facilitate the user to find the destination.

\subsection{Related algorithm design}

\subsubsection{Determine the navigation direction}

Use the vector cross product to determine whether it is counterclockwise or clockwise, so as to determine whether the navigation direction is left or right.

Let vector $\mathrm{p}=(\mathrm{x} 1, \mathrm{Y} 1), \mathrm{q}=(\mathrm{X} 2, \mathrm{Y} 2)$, and $\mathrm{r}=(\mathrm{X} 3, \mathrm{Y} 3)$; calculate $(\mathrm{x} 2-\mathrm{x} 1)(\mathrm{y} 3-\mathrm{y} 1)-(\mathrm{y} 2-\mathrm{y} 1)(\mathrm{x} 3-\mathrm{x} 1)$ according to vector $\mathrm{p}$, vector $\mathrm{q}$, and vector $\mathrm{r}$; then, determine the size of the result. If it is greater than 0 , turn clockwise to the right; if it is less than 0 , turn counterclockwise to the left; if it is equal to 0 , it is collinear, which means, it may be straight forward or backward.

\subsubsection{Obtain track coordinates}

As shown in Figure 1, assuming that people walk 1.4 meters per second, it is possible to determine the coordinates of $\mathrm{B}, \mathrm{C}$, and $\mathrm{P} 2$ according to each second. Then, using the principle of similar triangles, the coordinates of A can be determined.

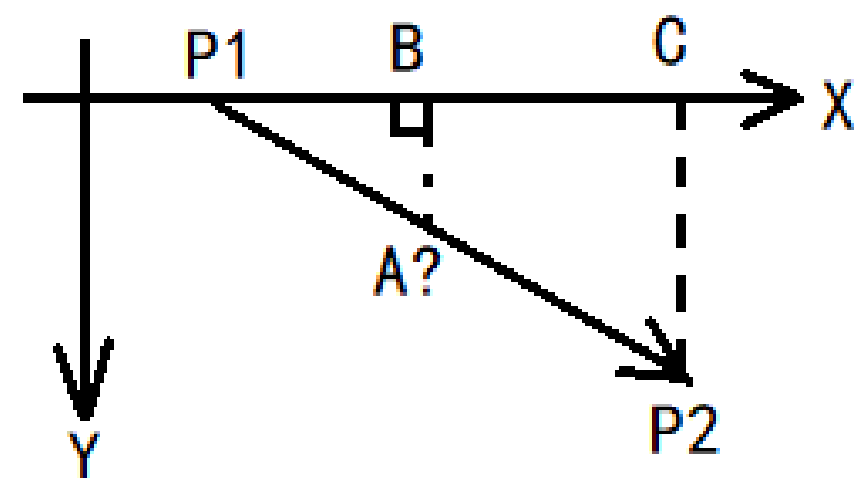

Figure 1. Obtaining the coordinates on the trajectory line

\section{Implementation of the system}

\subsection{Realization of user interaction function}

(1) Upon receiving the message sent by the user, call the encapsulated parseXML() method to parse the request message and obtain the message type MsgType to determine the message type sent by the user. Message types include subscribe, unsubscribe, click, etc.

(2) Determine whether the type of MsgType message is a push type event. If the two types are the same, continue to ascertain whether the event type is a subscribe type. If yes, push the text message to the user; if not, send an error message.

(3) If the event type is equal to the user-defined menu item click event, determine which option the user clicks according to the value of the key. If the event attribute key value is the same as the key value of the menu creation, create a graphic message, and reply the user; otherwise, send an error message.

(4) Finally, the message object is encapsulated and transformed into the corresponding XML format. 


\subsection{Realization of indoor positioning and navigation function}

(1) Realization of "location confirmation" function

After clicking "location confirmation," the user's current location information is determined, which can realize the scanning function. When the user clicks "get current location," the WeChat scanning interface function is called, and the text information is obtained from the QR code; that is, the user's current location information.

(2) Realization of "visit navigation" function

With the "visit navigation" function, the code can be scanned to confirm the departure, and the destination can then be entered to determine the starting point of the navigation. With that, the "visit navigation" function is realized, as shown in Figure 2.

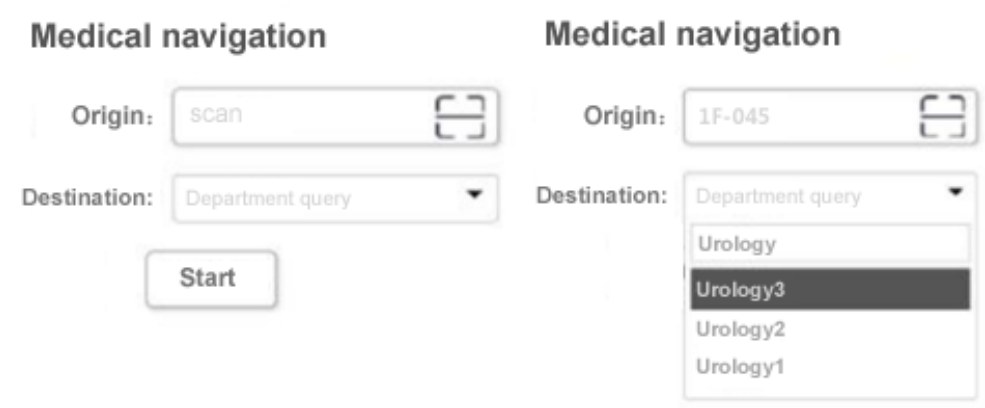

Figure 2. Visit navigation

The departure and destination in the navigation is from "wound care room" to "urology," respectively. The navigation diagram is shown in Figure 3.
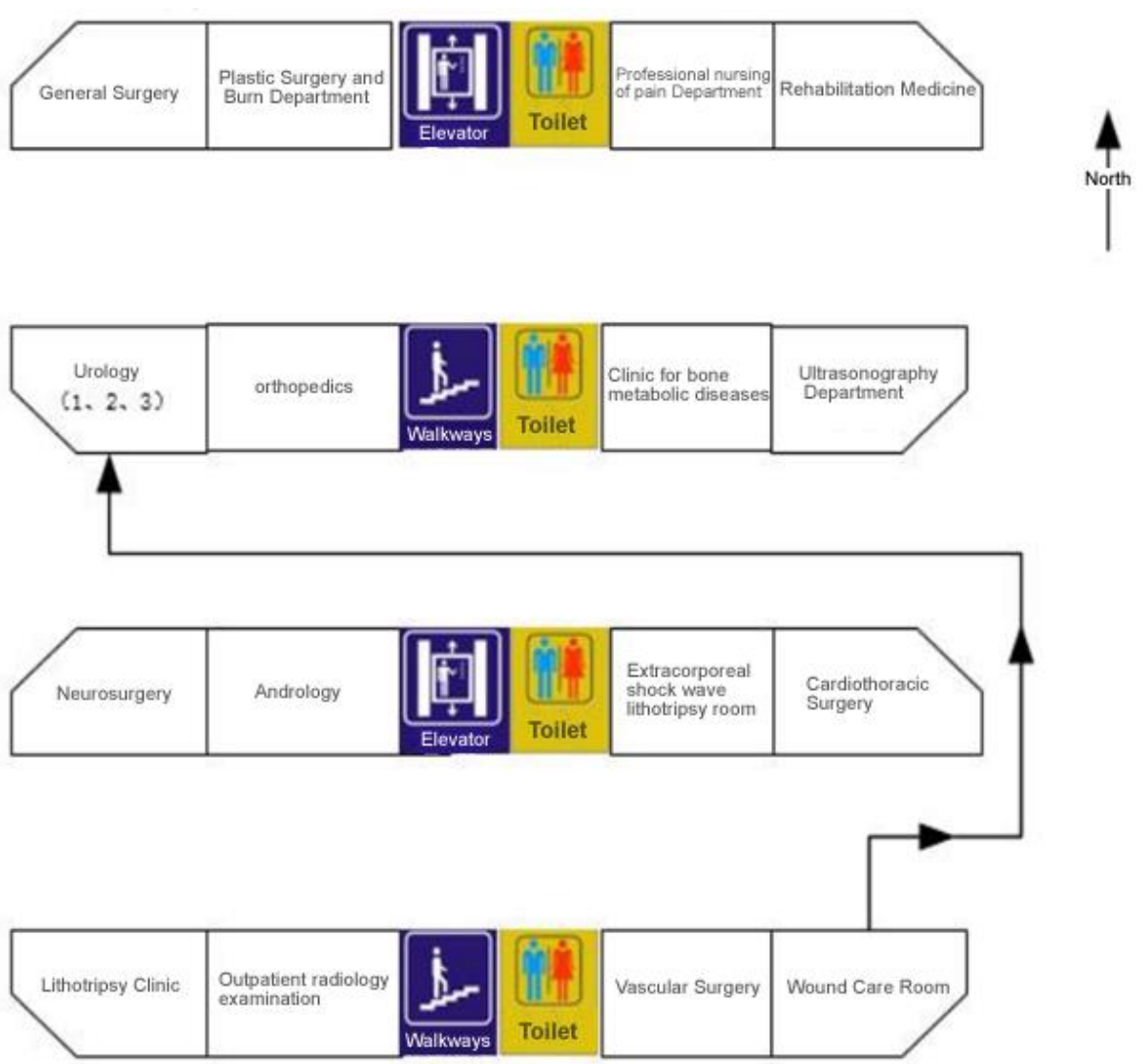

Figure 3. Navigation diagram 
(3) Realization of "floor guidance" function

This function is mainly used to guide users to obtain the overall layout information of the hospital. The building submenu can be clicked to push the specific department information of each floor of the selected department building to the user.

\section{Conclusion}

The hospital indoor navigation system based on WeChat can realize the indoor navigation between different departments in a hospital. Users can locate and navigate in real time through the QR code using their mobile phones and other handheld terminals, which is convenient to guide users to find relevant departments.

The core problem solved in this paper is the use of WeChat public platform to scan QR codes for location confirmation. Upon location confirmation and entering the destination, navigation will then be given to the user. Further research and design can be centered in this area in the future.

\section{Disclosure statement}

The author declares no conflict of interest.

\section{References}

[1] Wang B, Wang J, Yang X, et al., 2016, Design and Construction of Hospital Navigation System Based on QR Code Technology. People's Military Doctor, 2016(10): 1079-1080.

[2] Ge S, 2015, Development and Implementation of Patient Medical Service System Based on WeChat Platform, Nanjing Normal University. DOI: 10.7666/d.Y2857351

[3] Yang C, Zhang D, Li X, et al., 2016, Research on Hospital Department Positioning and Navigation System Based on QR Code. China Digital Medicine, 11(60): 58-60, 94. 